LAL 07-157

EUROTeV-Report-2007-017

October 2007

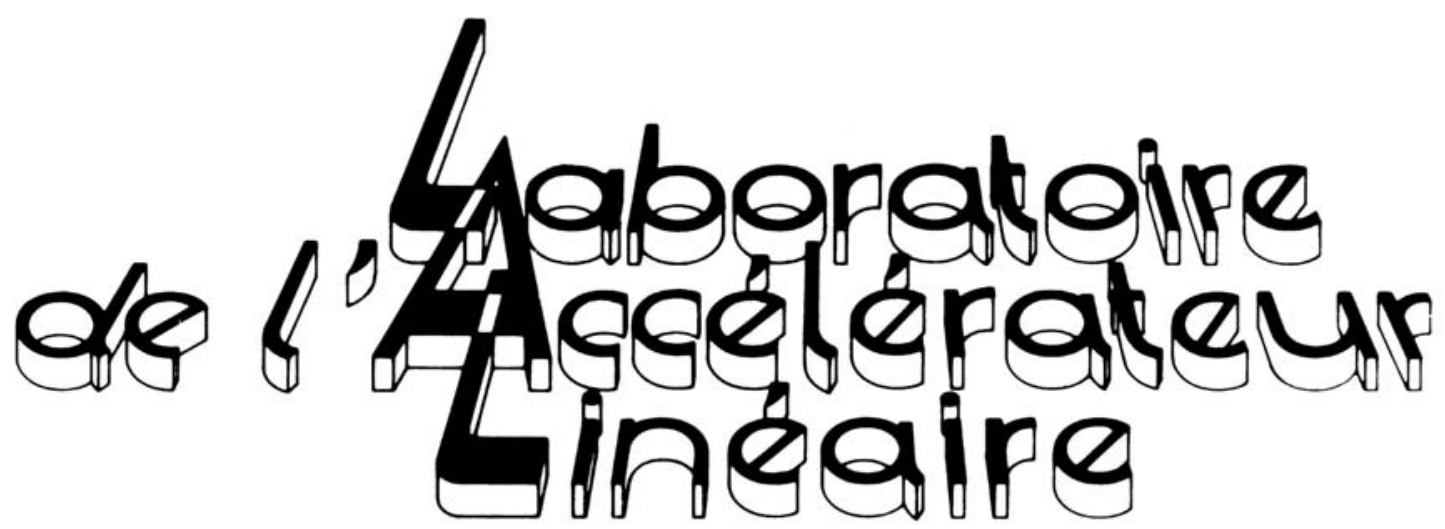

\title{
Impact of beam-beam effects on precision luminosity measurements at the ILC
}

\author{
C. Rimbault, P. Bambade
}

LAL, Univ Paris-Sud, IN2P3/CNRS, Orsay, France

K. Mönig

DESY, Zeuthen, Germany

D. Schulte

CERN, Geneva, Switzerland

U.M.R

de

l'Université Paris-Sud

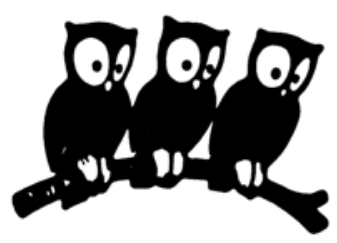

Institut National de Physique Nucléaire et de Physique des Particules du CNRS 


\title{
Impact of beam-beam effects on precision luminosity measurements at the ILC
}

\author{
C. Rimbault, P. Bambade*, K. Mönig ${ }^{\dagger}$, D. Schulte ${ }^{\ddagger}$
}

\begin{abstract}
In this paper, the impact of beam-beam effects on the precision luminosity measurement at the International Linear Collider is investigated quantitatively for the first time. GUINEA-PIG, a beam-beam interaction simulation tool, is adapted to treat the space charge effects affecting the Bhabha events used in this measurement. The biases due to the resulting changes in kinematics are evaluated for different center-of-mass energies and beam parameters.
\end{abstract}

${ }^{*}$ LAL, Univ Paris-Sud, IN2P3/CNRS, Orsay, France

${ }^{\dagger}$ DESY, Zeuthen, Germany

${ }^{\ddagger}$ CERN, Geneva, Switzerland 


\section{Introduction}

A way to determine luminosity at the International Linear Collider (ILC) is to measure the event rate of the Bhabha scattering process in a finely segmented calorimeter (LumiCal) at very low polar angles in the very forward region of the ILC detector. An absolute precision between $10^{-4}$ and $10^{-3}$ is needed for a number of key physics measurements $[1,2]$. Besides theoretical uncertainties on the cross section of the Bhabha process [3] and different experimental errors when identifying Bhabha events in the LumiCal [4], the very strong beam-beam space charge effects which characterise the ILC $e^{+} e^{-}$collisions can lead to large biases in the counting rate. These must be corrected accurately enough not to limit the precision of the luminosity measurement.

In this paper, Bhabha event samples produced with the BHLUMI event generator [5] are used in the context of the GUINEA-PIG beam-beam interaction simulation [6], to study how the experimental selections specified to determine the ILC luminosity are affected by beam-beam space charge effects. After recalling the basic principle of the luminosity measurement, the procedure developed to account for beamstrahlung radiation and electromagnetic deflections in the computation of the final state and the corresponding implementation in a modifed version of the GUINEA-PIG program are described. The magnitude of the different resulting biases and their dependencies on the choice of experimental selection cuts are then evaluated for the first time. A datadriven correction method to minimise the part of the bias induced by beamstrahlung is also suggested and discussed, based on determining the luminosity spectrum within the LumiCal. Finally, expected residual uncertainties after corrections are estimated for a representive set of beam parameters and centre-of-mass energies specified at the ILC.

\section{Input parameters and overview of procedure}

Experimentally, the integrated luminosity $\mathcal{L}_{\text {int }}$ of a $e^{+} e^{-}$collider such as the ILC can be measured by counting the number of Bhabha scattering events $N_{B h}$ within a given angular acceptance:

$$
\mathcal{L}_{\text {int }}=\frac{N_{B h}}{\sigma_{B h}},
$$

where $\sigma_{B h}$ is the cross section for the Bhabha process computed within this angular acceptance.

To study the biases induced by beam-beam space charge effects, a sample of Bhabha events is first produced with BHLUMI (version 4.04), a multiphoton Monte-Carlo event generator for small-angle Bhabha scattering including $\mathcal{O}(\alpha)$ radiative corrections [5] and providing four-momenta of outgoing electron, positron and photons. The center-of-mass energy is $500 \mathrm{GeV}$ and the scattering angles are generated in the range $25 \mathrm{mrad}<\theta<$ $90 \mathrm{mrad}$.

The four-momenta of the two charged final state particles of a generated event are then read into GUINEA-PIG and associated to one of the $e^{+} e^{-}$interactions occuring 
during the simulated bunch collision. An arbitrary event selection probability is used at this stage to obtain a final sample of desired size. This allows using GUINEA-PIG to compute the subsequent electromagnetic transport through the remaining part of the colliding bunch and to obtain the distribution of the resulting deflections with the appropriate luminosity weighting. To take into account beamstrahlung emission and electromagnetic deflections of the particles prior to the hard interaction, the generated Bhabha events must be rescaled and boosted from the centre-of-mass system of the considered $e^{+} e^{-}$interaction to the laboratory frame and an appropriate rotation of the coordinate system should be applied ${ }^{1}$. By applying such transformations, new fourmomenta are successively obtained for the final state particles and are used to quantify the impact of the different effects.

A basic experimental selection of Bhabha events is obtained by requiring the coincidence of an electron and a positron in a back-to-back topology, with polar angles within $30 \mathrm{mrad}<\theta<75 \mathrm{mrad}$ and energies larger than $0.8 E_{\text {beam. }}$. This basic selection is used in the studies presented below, along with an improved version optimised to reduce the biases from the studied space-charge effects (see Section 3.3.1). The Nominal ILC beam parameters shown in Table 1 were used for most results.

\begin{tabular}{cccccccc}
\hline \hline & $\begin{array}{c}E_{c m} \\
{[\mathrm{GeV}]}\end{array}$ & $\begin{array}{c}N \\
{\left[10^{10}\right]}\end{array}$ & $N_{b}$ & $\begin{array}{c}\beta_{x} / \beta_{y} \\
{[\mathrm{~mm}]}\end{array}$ & $\begin{array}{c}\gamma \epsilon_{x} / \gamma \epsilon_{y} \\
{\left[10^{-6} \mathrm{~m} . \mathrm{rad}\right]}\end{array}$ & $\begin{array}{c}\sigma_{x} / \sigma_{y} \\
{[\mathrm{~nm}]}\end{array}$ & $\begin{array}{c}\sigma_{z} \\
{[\mu \mathrm{m}]}\end{array}$ \\
\hline Nominal & 500 & 2 & 2820 & $21.0 / 0.4$ & $10.0 / 0.040$ & $655 / 5.7$ & 300 \\
Low Power & 500 & 2 & 1330 & $10.0 / 0.2$ & $10.0 / 0.035$ & $452 / 3.8$ & 200 \\
\hline \hline
\end{tabular}

Table 1: Nominal and Low Power ILC beam parameters. $E_{c m}$ is the center-of-mass energy of the collision, $N$ the number of particles per bunch, $N_{b}$ the number of bunches per train, $\beta_{x, y}$ the beta functions at the interaction point, $\gamma \epsilon_{x, y}$ the normalised emittances and $\sigma_{x, y, z}$ the RMS bunch dimensions at the interaction point.

\section{Effect of Beam-Beam Space Charge on Bhabha Scattering}

The cross section of the Bhabha process is given to first order by:

$$
\frac{d \sigma_{B h}}{d \theta}=\frac{2 \pi \alpha^{2}}{s} \frac{\sin \theta}{\sin ^{4} \theta / 2} \equiv \frac{32 \pi \alpha^{2}}{s} \frac{1}{\theta^{3}}
$$

where $\alpha$ is the fine-structure constant, $s$ the center-of-mass energy squared and $\theta$ the scattering angle. In the angular range between 30 and $75 \mathrm{mrad}$, this cross section

\footnotetext{
${ }^{1}$ The treatment assumes that the hard Bhabha process can be factorised from the softer interactions in the space charge of the bunch.
} 
amounts to approximately $4 \mathrm{nb}$ at $500 \mathrm{GeV}$, corresponding to about $4.5 \cdot 10^{-3}$ event per bunch crossing. It is shown as a function of $\theta$ in Figure 1.

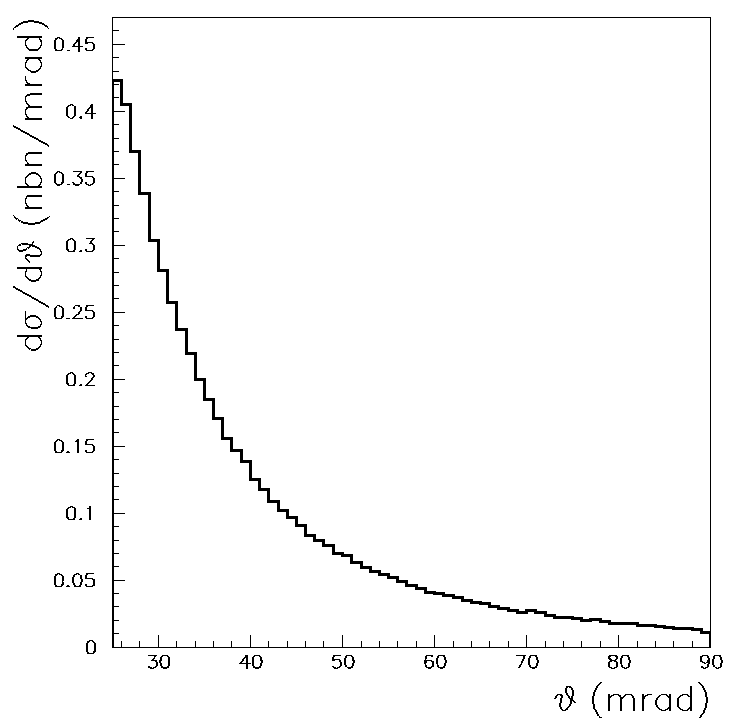

Figure 1: Cross section of the Bhabha process as a function of polar angle obtained from the BHLUMI [5] generation.

\subsection{Beamstrahlung Radiation}

Prior to the hard Bhabha scattering, the interacting particles are likely to have been deflected by the space charge of the opposite bunch and their energies reduced due to the emission of beamstrahlung. To take into account the cross section dependence with $s$, the probability used to produce Bhabha scattering events during the beam-beam collision is rescaled by $s / s^{\prime}$, where $s^{\prime}$ is the effective centre-of-mass energy (shown in Figure 2). The four-vectors of the Bhabha event particles are also rescaled by $\sqrt{s^{\prime} / s}$, to satisfy energy and momentum conservation, as well as boosted from the centre-of-mass system of the two interacting particles to the laboratory frame. Finally the coordinate system is rotated to take into account the deflection angles of the interaction particles in the initial state.

Just like the initial state radiation present in the initial sample generated with BHLUMI, the beamstrahlung emissions often occur asymmetrically, with either the electron or the positron loosing most of the energy. Hence the acollinearity of the final state can be significantly enhanced. This is illustrated in Figure 3, where the distributions of the polar angles of the two final state particles are shown in the range of the LumiCal acceptance, both for the initial sample generated with BHLUMI and after the boosting procedure. As can be seen, this enhances the acollinearity such that many of the events, initially within the defined angular acceptance of 30-75 mrad, corresponding to the basic selection cuts, 


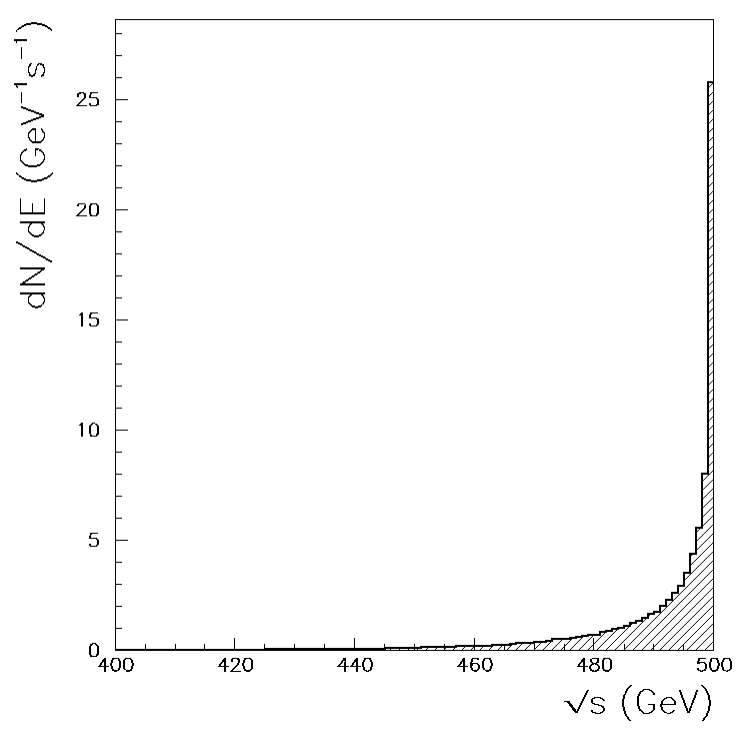

Figure 2: Effective center-of-mass energy spectrum of the scattering.

migrate outside, hence significantly reducing the counting rate. This reduction is only due to energy losses. For ideal bunches colliding head-on, the rotations of the coordinate system applied to the final state particles produce negligible effects on counting rates.

\subsection{Electromagnetic Deflections}

Given the transverse and longitudinal bunch sizes at the ILC (see Table 1), the final state particles scattered in the acceptance of the LumiCal following a Bhabha interaction can typically cross a significant part of the opposite bunch. They can thus be focused by the electromagnetic field from the corresponding space charge. In the GUINEAPIG simulation, the existing procedure to track secondary charged particles can be used conveniently to predict both the final deflection angle and any additional radiation. The resulting changes in scattering angles are displayed in Figure 4, where the differences between the initial polar angles before including the electromagnetic deflection, $\theta_{1}$ and the final ones including it, $\theta_{2}$ are shown as a function of $\theta_{1}$. The typical magnitudes of the induced electromagnetic deflections are a few $10^{-2} \mathrm{mrad}$, with the largest values at the lower edge of the LumiCal acceptance. Small energy losses due to radiation are also found.

\subsection{Impact on Bhabha Counting Rate in the LumiCal}

Both the beamstrahlung radiation and electromagnetic deflection effects described above lead to a suppression of the Bhabha counting rate in the defined experimental acceptance in comparison to the theoretically predicted one. This BHabha Suppression Effect (BHSE) can be expressed as: 

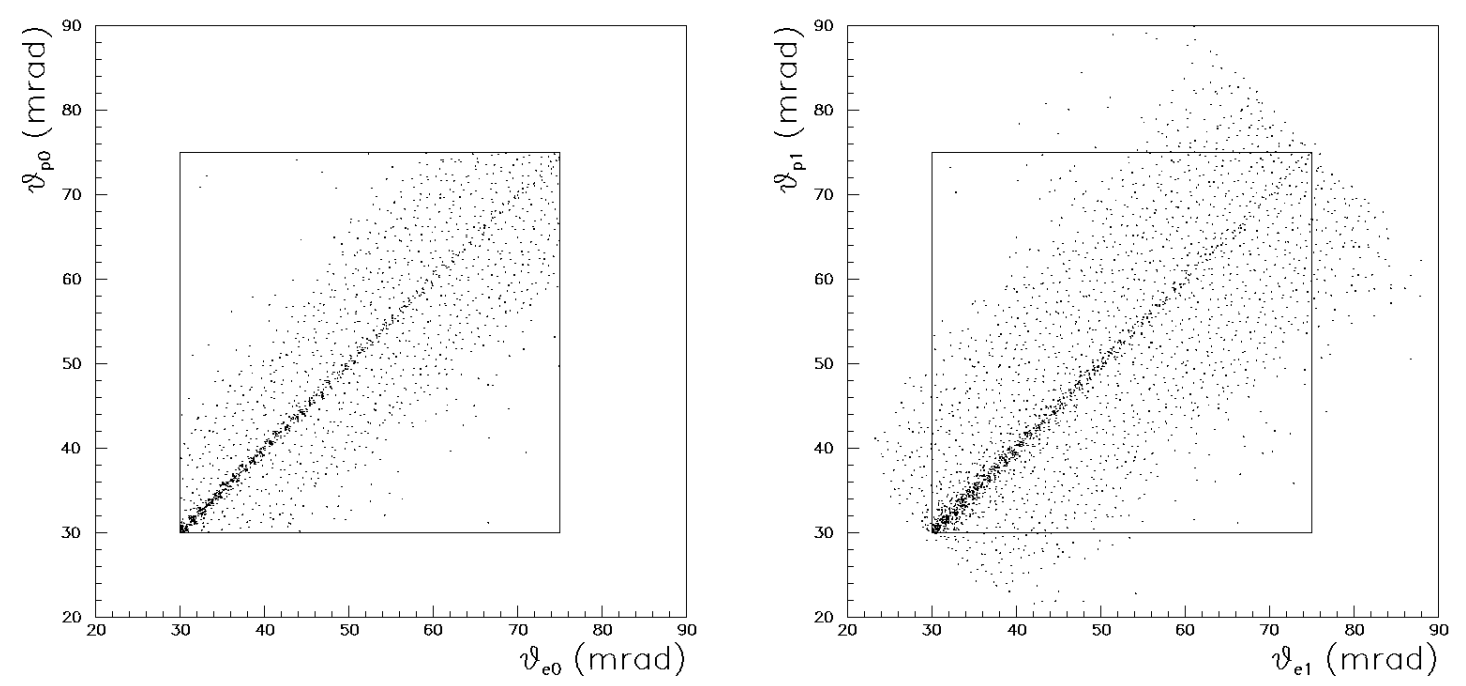

Figure 3: Polar angles of the final state positrons versus the electrons which were initially within the acceptance cuts specified in Section 2 (left) and their value after accounting for beamstrahlung radiation of the interacting particles in the initial state (right).

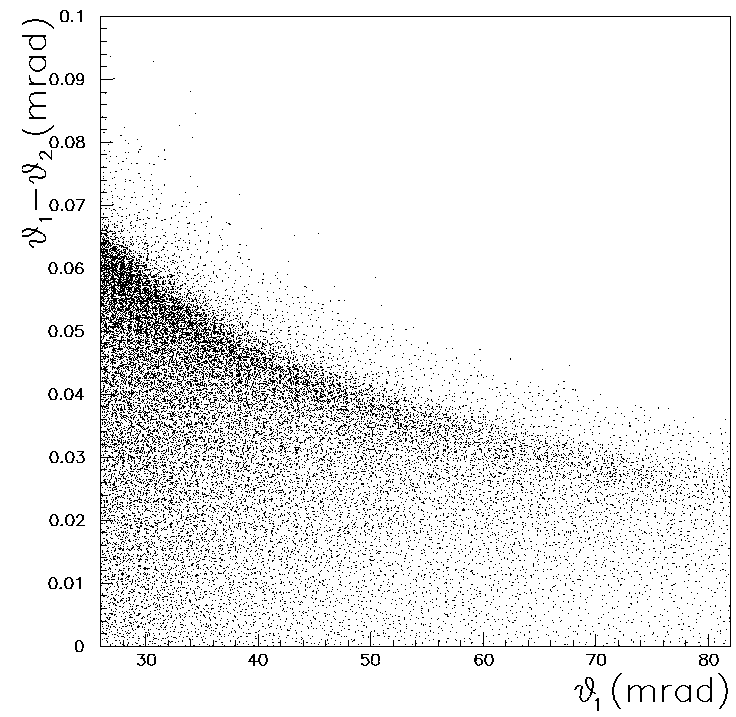

Figure 4: Change in Bhabha scattering final state polar angle due to the deflection induced by the space charge of the opposite bunch as a function of the polar angle at production. 


$$
\text { BHSE }=\frac{N_{\text {final }}-N_{\text {init }}}{N_{\text {init }}},
$$

where $N_{\text {init }}$ and $N_{\text {final }}$ are the numbers of Bhabha events selected within the specified cuts, respectively before and after including the different transformations to take into account effects from the beam space charge.

The magnitude of BHSE is shown in Table 2 for each effect, using the set of basic cuts listed in Section 2. The resulting bias on the Bhabha counting rate, and thus on the integrated luminosity measurement, is of the order of $-4.5 \%$. This large effect is sensitive to beam parameters and must be corrected through an experimental procedure allowing to adequately probe and monitor the different beam-beam effects. It should be noted that this is very different from the case of the bias induced by quantum electrodynamical effects such as final and initial state radiation, which can be computed theoretically with good accuracy, for example in the context of event generators such as BHLUMI[3].

Before discussing a data-driven correction method aimed at the larger of the two effects in Table 2 (arising from beamstrahlung) and before evaluating the dependence on beam parameters and the residual sensitivities which can be expected, it is shown below how the BHSE can be reduced using more convenient selection cuts.

\begin{tabular}{cc}
\hline \hline Nature of Effect & BHSE [\%] \\
\hline Beamstrahlung & $-3.78 \pm 0.04$ \\
Electromagnetic Deflections & $-0.65 \pm 0.02$ \\
\hline Total & $-4.41 \pm 0.05$ \\
\hline \hline
\end{tabular}

Table 2: Contributions to BHabha Suppression Effect (BHSE) from the different sources discussed (see text), computed within an angular acceptance from 30 to $75 \mathrm{mrad}$ and requiring each scattered particle to have more than $80 \%$ of the beam energy.

\subsubsection{Improved Event Selection Procedure}

Beamstrahlung radiation emitted prior to the hard Bhabha scattering enhances the acollinearity between the two final state charged particles. The experimental acceptance of the LumiCal, on the other hand, naturally favours collinear events when defined with symmetrical angular cuts. For this reason, asymmetrical angular cuts, first introduced by the LEP experiments $[7,8,9,10]$ and later adapted for ILC [4] are advantageous to minimise the BHSE and the resulting biases.

In the same line of thought, applying identical energy cuts to both final state charged particles is non-optimal since, as already noted, beamstrahlung emission prior to the hard scattering often occurs asymmetrically. The sensitivity of the different contributions to the BHSE to the energy cut is illustrated in Figure 5. As can be seen, mostly the 
contribution from beamstrahlung is affected: setting the cut at $80 \%$ of the beam energy multiplies it by more than a factor five as compared to only applying angular cuts, while it doubles the contribution from electromagnetic deflections. The advantage of using asymmetrical cuts also for the energies is best illustrated in the two-dimensional scatter plot in Figure 6, where one can see that it is more natural to choose a global energy cut based on the sum of the final state energies rather than individual ones.

As an illustration of the reduction in sensitivity which can be achieved, the following asymmetrical selection cuts for the angles and energies of the final state particles were applied:

$$
\begin{gathered}
30 \mathrm{mrad}<\theta_{+/-}<75 \mathrm{mrad} \text { and } 26.2 \mathrm{mrad}<\theta_{-/+}<82 \mathrm{mrad}^{2} \\
E_{-}+E_{+}>0.8 \sqrt{\mathrm{s}},
\end{gathered}
$$

where the two sets of angular cuts are applied randomly, respectively to either the electron and positron, or vice-versa. As shown in Table 3, applying these new cuts reduces the total Bhabha suppression effect by about a factor three.

\begin{tabular}{cc}
\hline \hline Nature of Effect & BHSE [\%] \\
\hline Beamstrahlung & $-1.03 \pm 0.04$ \\
Electromagnetic Deflections & $-0.48 \pm 0.02$ \\
\hline Total & $-1.51 \pm 0.05$ \\
\hline \hline
\end{tabular}

Table 3: Contributions to BHabha Suppression Effect (BHSE) from the different sources discussed (see text), computed with the asymmetrical selection cuts for the final state particle angles and energies specified in Equations 4 and 5.

\section{Luminosity Spectrum Reconstruction}

In this section, a data-driven correction method is suggested for the main component of the bias induced on the Bhabha counting rate by beam-beam effects, i.e. that which arises from the radiation of beamstrahlung by the interacting particles prior to the hard scattering (see Tables 2 and 3). This method is based on reconstructing the luminosity spectrum from the measurement of the polar angles of the final state charged particles. Using energy-momentum conservation, it can be shown, in the assumption that all the radiation is collinear to the beam and emitted in the same direction, that [11]:

$$
\frac{\sqrt{s^{\prime}}}{\sqrt{s}} \simeq \sqrt{1-2 \frac{\sin \left(\theta_{1}+\theta_{2}\right)}{\sin \left(\theta_{1}+\theta_{2}\right)-\sin \theta_{1}-\sin \theta_{2}}},
$$




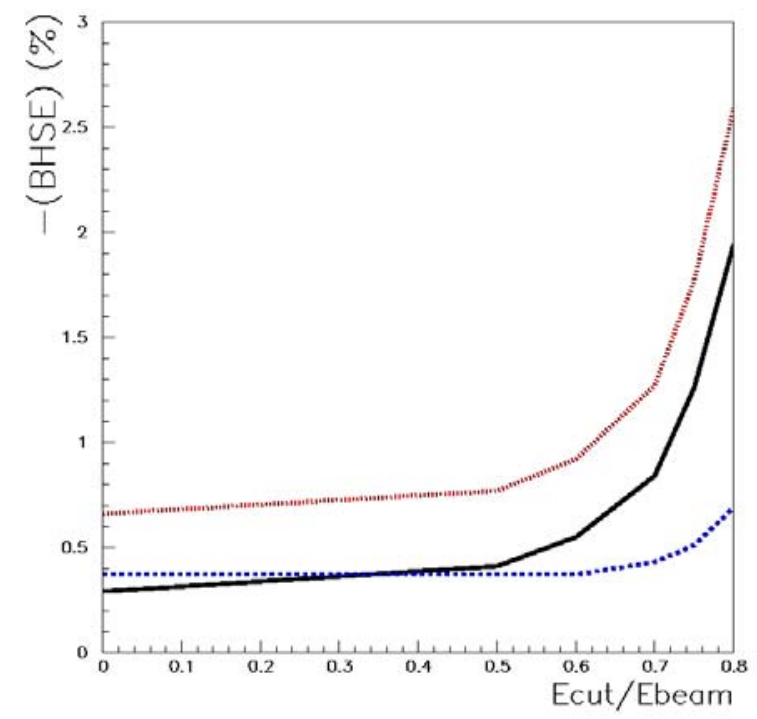

Figure 5: Absolute value of BHabha Suppression Effect (BHSE) as a function of the cut on each of the energies of the final state particles, normalised to the beam energy. The effects are shown separately after solely modifying the initial state prior to the Bhabha scattering (continuous line), solely implementing post-scattering electromagnetic deflections (dashed line) and for both effects combined (top dotted line).

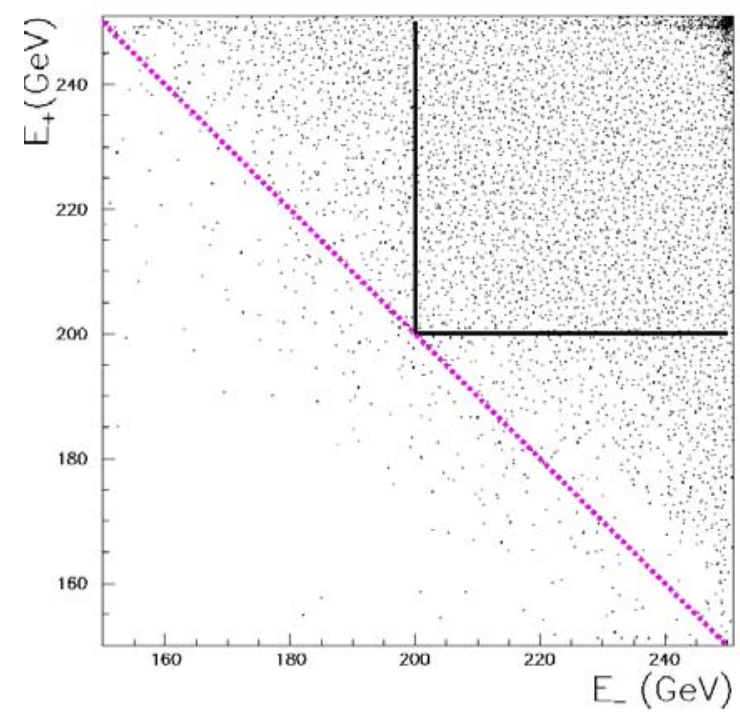

Figure 6: Energy of the positron versus energy of the electron for each the Bhabha events passing the angular cuts defined in Equation 4. 
where $\sqrt{s}$ and $\sqrt{s^{\prime}}$ are, respectively, the nominal and effective centre-of-mass energies and $\theta_{1,2}$ are the angles defined relatively to the direction of the emitted photons (such that $\left.\theta_{1}+\theta_{2}>\pi\right)$.

The left-hand side of Equation 6 is calculable theoretically from the four-momenta within the simulation while the right-hand side is accessible experimentally from the angles measured in the LumiCal. Both are referred to below respectively as $x_{t h}$ and $x_{\text {rec }}$. Both quantities are shown in Figure 7 , at the different stages of the treatment. It is noteworthy that the reconstructed luminosity spectrum is almost not modified by the electromagnetic deflections, while the effect from beamstrahlung is as expected clearly visible.

This suggests that reconstructing the luminosity spectrum in this way enables measuring the amount of beamstrahlung emitted and infering a correction for the corresponding part of the BHSE, independent of the electromagnetic deflections. However, it also means that this method does not allow probing electromagnetic deflections experimentally. In the following, the accuracy with which the luminosity spectrum should be measured for a given luminosity precision is considered to illustrate the potential of the method.
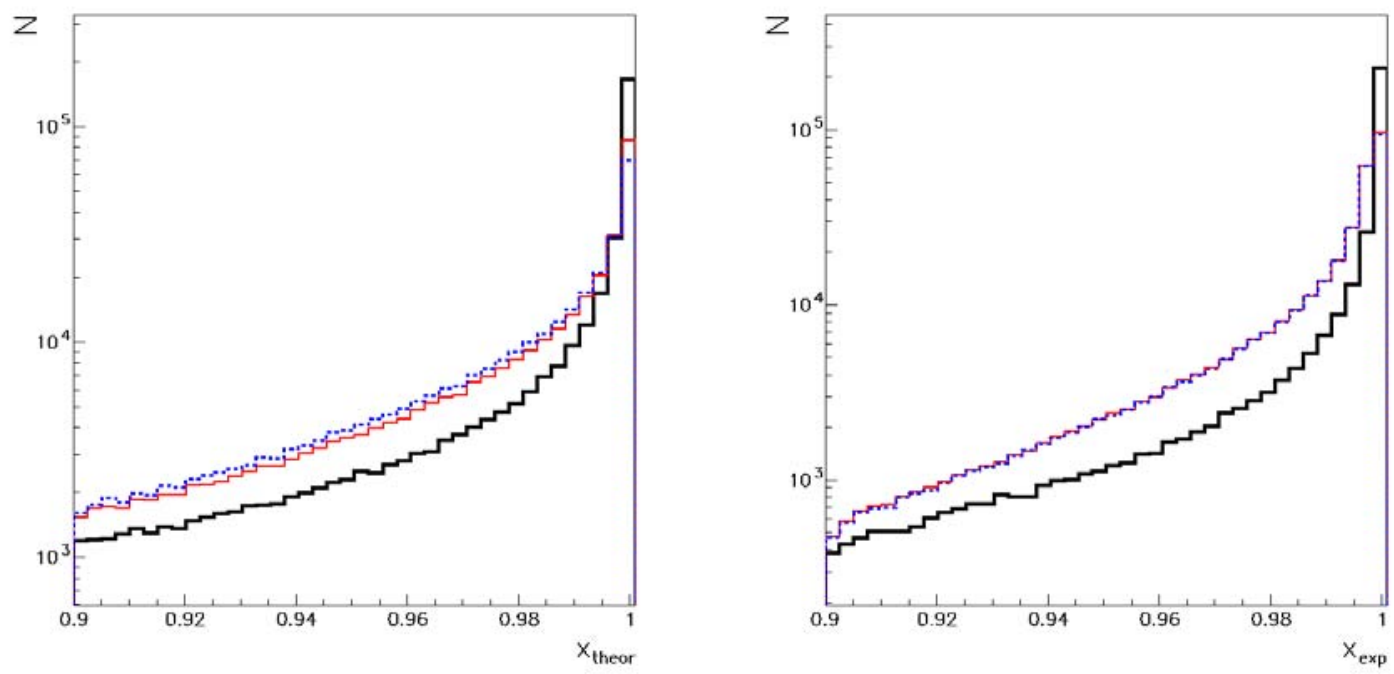

Figure 7: Theoretical (left) and experimental (right) ratios of luminosity spectrum for the differents stages in the treatment of Bhabha events to take into account beam-beam effects: initial sample from the BHLUMI generator, with initial state radiation but without any beam-beam effects (continuous thick line), after including beamstrahlung radiation and rotation of the coordinate system prior to the hard scattering (continuous thin line) and after including also post-scattering electromagnetic deflections (dashed line). 


\subsection{Expected Reconstruction Accuracy}

A description of the on-going R\&D on Forward Calorimeters at the ILC can be found in the reference [2]. Depending on the readout technology, polar angles are expected to be measured with an error $\sigma_{\theta}$ between $0.031 \mathrm{mrad}$ and $0.13 \mathrm{mrad}$. The sensitivity of the luminosity spectrum measurement was estimated by adding a Gaussian error to the polar angle of each final state particle used in the reconstruction, using the larger of these two values. The result is shown in Figure 8.

It was found that a $\sigma_{\theta}$ of $0.13 \mathrm{mrad}$ induces only a small error on the luminosity spectrum reconstruction, with its mean value, $\left\langle x_{\text {rec }}\right\rangle$, being shifted by only $5 \cdot 10^{-4}$.

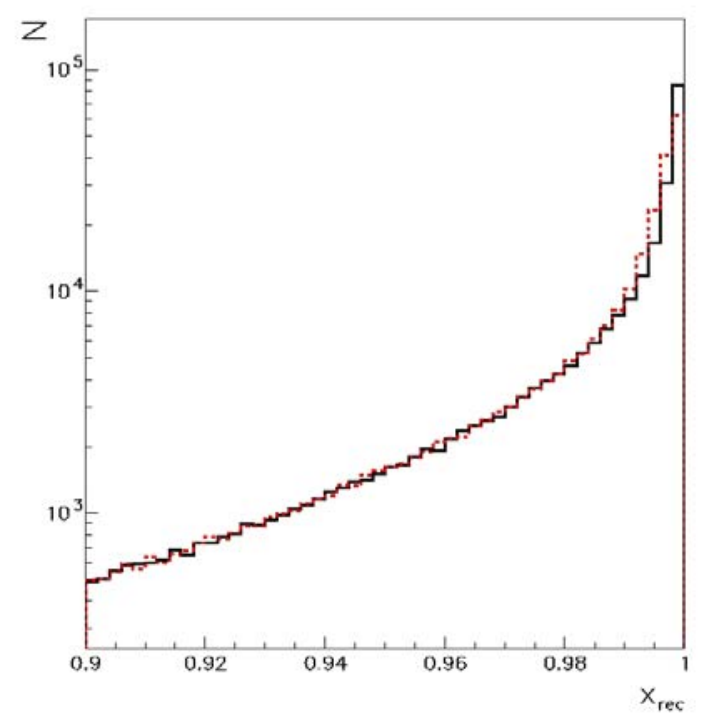

Figure 8: Reconstructed luminosity spectrum from final state particle polar angles, with (continuous line) and without (dashed line) adding a Gaussian error with $\sigma_{\theta}=$ $0.13 \mathrm{mrad}$.

\subsection{Required Reconstruction Accuracy to Control the BHSE}

The magnitude of the bias on the luminosity measurement induced by beam-beam effects depends on the amount of beamstrahlung emitted during collisions, which in turn can be expected to vary with beam parameters. The luminosity spectrum, which can be reconstructed by measuring the Bhabha events in the LumiCal, is directly related to the beamstrahlung and can be used to monitor the variations. The precision with which the luminosity spectrum (or its mean value) can be reconstructed should be compared to the expected impact of typical beam parameter variations.

To obtain an estimate in a simple way, two simulations were performed modifying the widths of the colliding bunches from the Nominal value listed in Table 1 to $\sigma_{x}=555 \mathrm{~nm}$ and $\sigma_{x}=755 \mathrm{~nm}$. The corresponding reconstructed luminosity spectra are displayed 


\begin{tabular}{cccc}
\hline \hline$\sigma_{x}[\mathrm{~nm}]$ & $\mathcal{L}\left[10^{34} \mathrm{~cm}^{-2} s-1\right]$ & $\left\langle x_{\text {rec }}>\right.$ & BHSE [\%] \\
\hline 555 & 2.5 & 0.976 & -2.22 \\
755 & 1.7 & 0.980 & -1.14 \\
\hline$\Delta$ & & $4 \cdot 10^{-3}$ & $10^{-2}$ \\
\hline \hline
\end{tabular}

Table 4: Luminosity $\mathcal{L}$, reconstructed luminosity spectrum mean value $\left\langle x_{\text {rec }}\right\rangle$ and magnitude of BHSE for two values of the colliding bunch width, $\sigma_{x} . \Delta$ is the difference between the values found for the two bunch widths. The samples used correspond approximately to a luminosity of $0.1 \mathrm{fb}^{-1}$. The associated statistical uncertainty on the mean value of the luminosity spectrum reconstruction within the cuts of the analysis is about $6 \cdot 10^{-5}$.

in Figure 9 and the impact of these changes on the luminosity, on the average of the luminosity spectrum and on the BHSE are listed in Table 4. From the values listed can be inferred that to maintain the bias on the integrated luminosity measurement below $10^{-3}$, variations in the reconstructed luminosity spectrum need to be known with a precision of $4 \cdot 10^{-4}$. This is reasonably well matched to the expected reconstruction accuracy of $5 \cdot 10^{-4}$ estimated above for this measurement. Improved sensitivity to beam parameter variations could also in principle be obtained by fitting the shapes of the different luminosity spectra [11]. Moreover the detector resolutions will in practice also be known at some level, which can improve the accuracy of the luminosity reconstruction.

\section{Sensitivity to beam parameters}

\subsection{Varying spot sizes and offsets at the interaction point}

In this section, the sensitivity of the BHSE to bunch parameters at the collision point and the residual dependencies which can be expected after correcting for the main contribution arising from beamstrahlung (emitted by the interacting particles prior to the Bhabha scattering) are discussed.

An example worth highlighting is the proposed Low Power beam parameters listed in Table 1. The BHSE is much more important in this case compared to Nominal parameters (see Table 3), reaching $-6.70 \%$ for the combined effects of beamstrahlung $(-5.51 \%)$ and electromagnetic deflections $(-1.25 \%)$, after applying the improved selection cuts described in Section 3.3.1.

During operation of the ILC, bunch sizes and alignment at the interaction point can be expected to vary over time, due to dynamical imperfections in the acceleration and optical transport and through injection errors. Dedicated feedback control loops are included in the ILC design to maintain the bunch parameters constant within appropriate tolerances [12]. In this context, it is interesting to evaluate the precision needed on the 


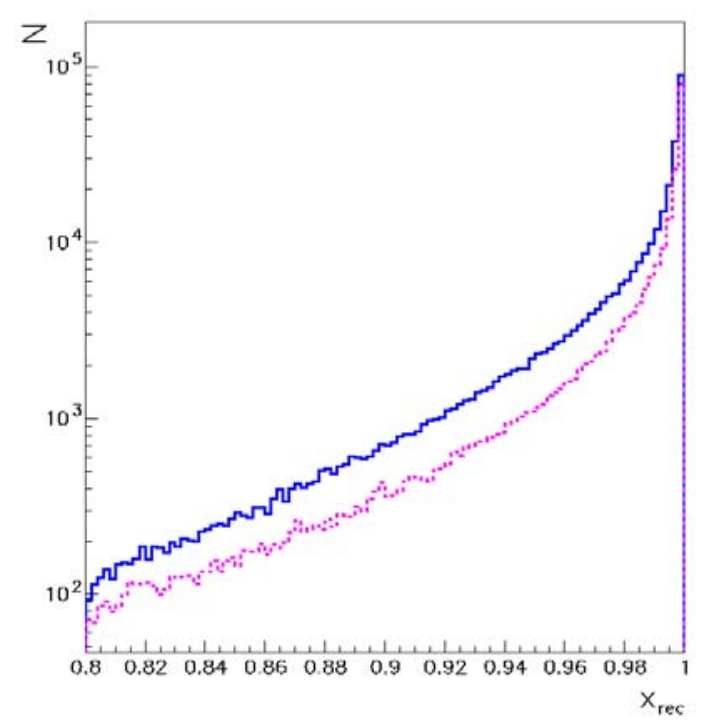

Figure 9: Reconstructed luminosity spectra from the final state particle polar angles, considering colliding bunch widths of $555 \mathrm{~nm}$ (continuous line) and $755 \mathrm{~nm}$ (dashed line).

knowledge of the main parameters to keep the BHSE within a given level of accuracy. Below, the variation of the different components of the BHSE is shown when changing one bunch parameter at the time and keeping the others fixed.

\subsubsection{Transverse bunch offsets and vertical size at the interaction point}

Transverse offsets at the interaction point lead to minor changes in the BHSE. This relative insensitivity is illustrated in the left-hand side of the Figure 10 for offsets up to 1.5 times the transverse bunch size, covering the range over which a useful luminosity can be produced. Similarly, the BHSE is rather insensitive to the vertical size $\sigma_{y}$ of the bunches. This is shown in the right-hand side of the Figure 10. Finally it was also checked that the BHSE is insensitive to longitudinal shifts of the beam waist.

\subsubsection{Bunch length and horizontal size at the interaction point}

On the other hand, the BHSE has a strong dependence on both the bunch length $\sigma_{z}$ and horizontal size $\sigma_{x}$, as those parameters are directly related to the beamstrahlung emission. The behaviour is shown in Figure 11. From the non-linear curves can be estimated the uncertainty on the BHSE resulting from a given precision assumed in the knowledge of $\sigma_{z}$ and $\sigma_{x}$. The values obtained are displayed in Table 5, both separately for the two contributions to the BHSE, and globally. It should be noted that the two effects can be added up independently, since most events radiate very little energy and 

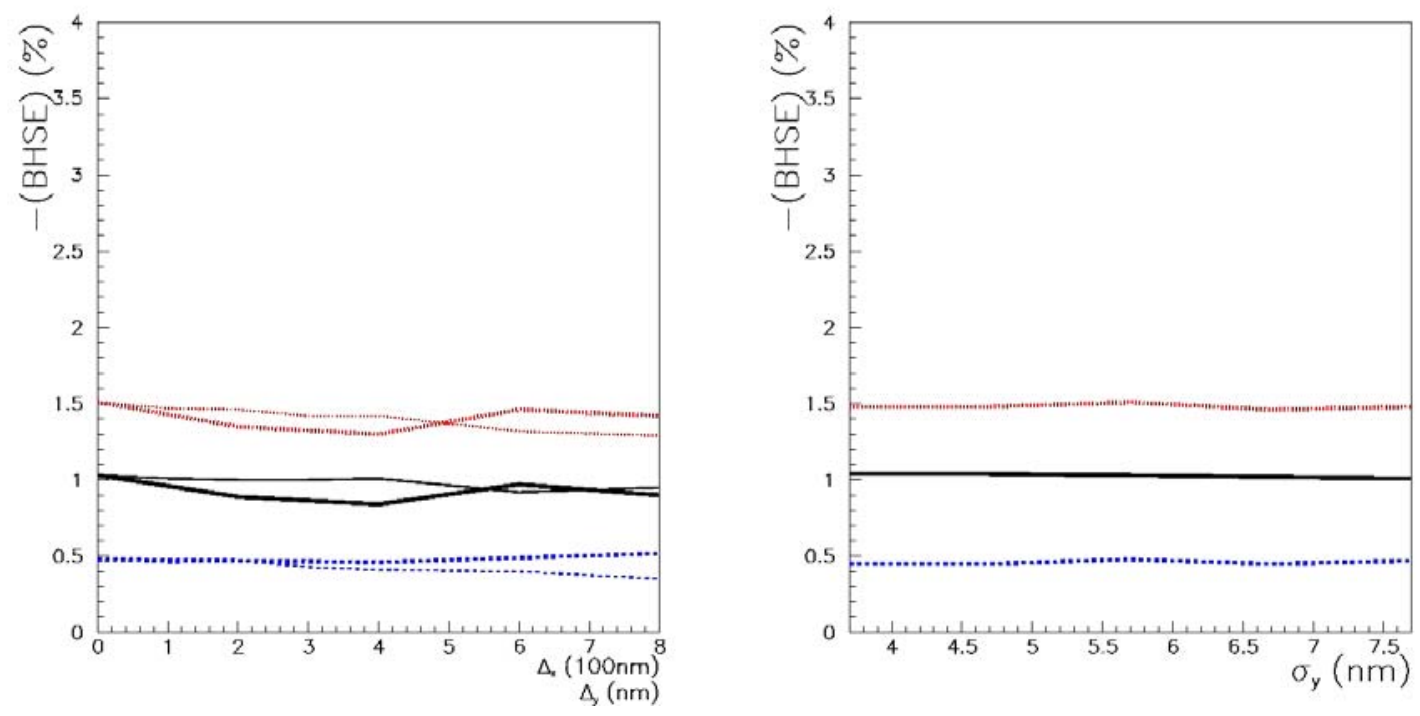

Figure 10: Bhabha suppression effect as function of horizontal (thin lines) and vertical (thick lines) offsets of the beams at the interaction point (left) and of the vertical bunch size (right). The contributions due to beamstrahlung (full line) and electromagnetic deflections (dashed line) are shown as well as the combined effect (top dotted line).

are hence deflected similarly. This can be seen clearly in Figure 11, where the global effect is the sum of the two contributions over the whole range.

The main uncertainty on the BHSE arises through the contribution from beamstrahlung. This part can in principle be measured and corrected from the luminosity spectrum reconstruction described in Section 4. The residual uncertainty which remains after such a procedure comes from the contributions of electromagnetic deflections. As an example, to limit the error on the BHSE from this part to about $10^{-3}$, a precision at the $20 \%$ level is needed on the knowledge of $\sigma_{z}$ and $\sigma_{x}$. It is worth noticing that although the magnitude of the dependence with $\sigma_{x}$ and $\sigma_{z}$ of each of the contribution of the BHSE is not the same, some bounds can still be inferred for the contribution from electromagnetic deflections, since both effects go in the same direction.

\subsection{Energy dependence}

ILC should allow physics runs initially for energies between the Z boson mass and $\sqrt{s}=$ $500 \mathrm{GeV}$ [13]. In this energy range beam-beam effects are strongly modified. For a flat beam, the mean relative beam energy loss due to beamstrahlung, $\delta$, can be written:

$$
\delta \propto \frac{N^{2} \gamma}{\sigma_{x}^{2} \sigma_{z}} \propto \frac{N^{2} \gamma^{2}}{\epsilon_{x}^{*} \beta_{x} \sigma_{z}} .
$$



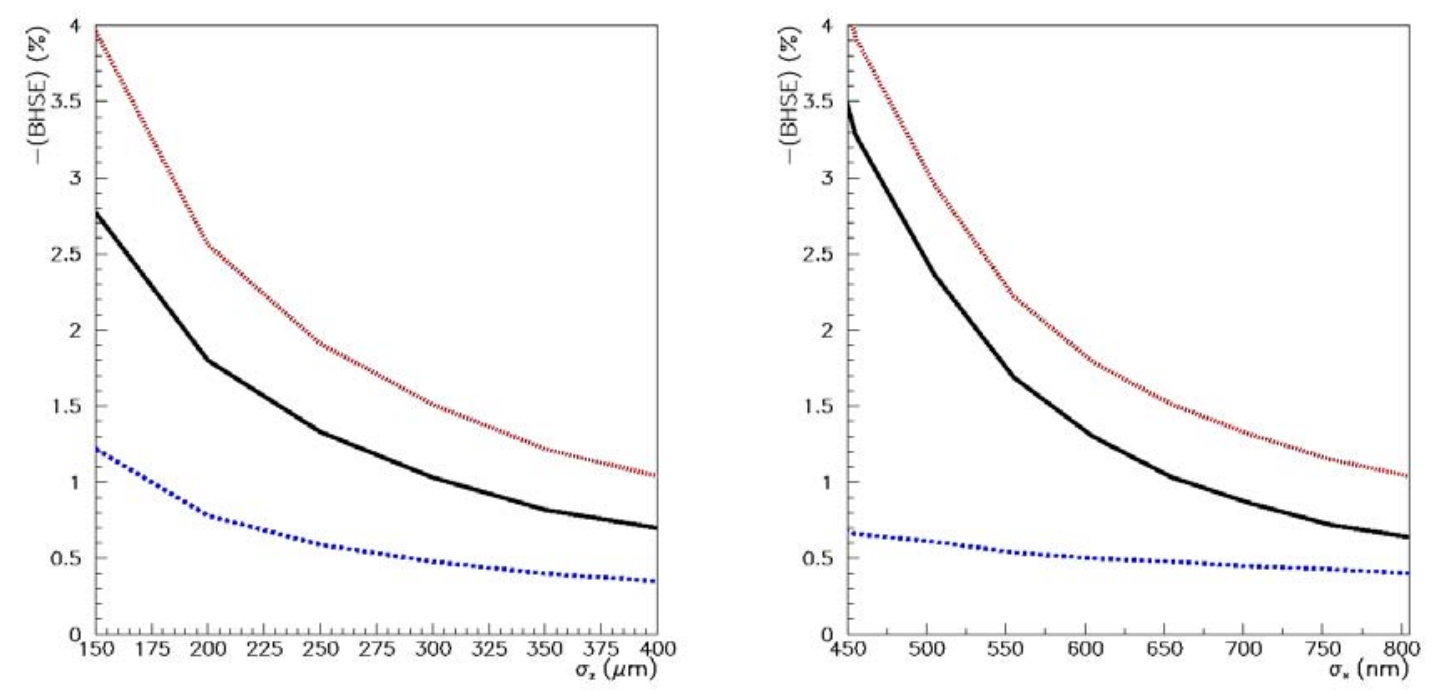

Figure 11: Bhabha suppression effect as a function of $\sigma_{z}$ (left) and $\sigma_{x}$ (right). The contributions due to beamstrahlung (full line) and electromagnetic deflections (dashed line) are shown as well as the combined effect (top dotted line).

\begin{tabular}{cccc}
\hline \hline$\frac{\Delta \sigma_{z}}{\sigma_{z}}$ & $\Delta B H S E_{B S}[\%]$ & $\Delta B H S E_{E M}[\%]$ & $\Delta B H S E[\%]$ \\
\hline $20 \%$ & -0.40 & -0.15 & -0.50 \\
& +0.25 & +0.10 & +0.30 \\
\hline $10 \%$ & -0.20 & -0.07 & -0.25 \\
& +0.15 & +0.05 & +0.15 \\
\hline $5 \%$ & -0.10 & -0.03 & -0.15 \\
& +0.05 & +0.02 & +0.05 \\
\hline \hline$\frac{\Delta \sigma_{x}}{\sigma_{x}}$ & $\Delta B H S E_{B S}[\%]$ & $\Delta B H S E_{E M}[\%]$ & $\Delta B H S E[\%]$ \\
\hline $20 \%$ & -1.10 & -0.10 & -1.20 \\
& +0.35 & +0.08 & +0.40 \\
\hline $10 \%$ & -0.40 & -0.04 & -0.45 \\
& +0.20 & +0.04 & +0.25 \\
\hline $5 \%$ & -0.20 & -0.02 & -0.20 \\
& +0.10 & +0.02 & +0.10 \\
\hline \hline
\end{tabular}

Table 5: Absolute errors on the BHSE due to increasing levels of uncertainty in the bunch length $\sigma_{z}$ and horizontal size $\sigma_{x}$. Statistical errors (not shown) are similar as in Table 3. The contributions from beamstrahlung and electromagnetic deflections, labelled respectively BS and EM, are shown separately in the second and third columns. 
The luminosity by bunch crossing can be expressed conveniently as:

$$
\mathcal{L}=\frac{N^{2}}{4 \pi \sigma_{x} \sigma_{y}} H_{D}=\frac{N^{2} \gamma}{4 \pi \sqrt{\epsilon_{x}^{*} \beta_{x} \epsilon_{y}^{*} \beta_{y}}} H_{D} \propto \sqrt{\frac{\delta}{\epsilon_{y}}} \frac{P_{\text {beam }}}{E} H_{D},
$$

where $N$ is the number of particles per bunch, $H_{D}$ the disruption parameter, $\epsilon_{x, y}^{*}=\gamma \epsilon_{x, y}$ are the normalised emittances, $\gamma$ the Lorentz factor, $P_{\text {beam }}$ the beam power and where $\beta_{y}=\sigma_{z}$ has been assumed in the derivation of the last expression [6]. The dependence of BHSE with energy is shown on the left-hand side of Figure 12, in the assumption that all optical parameters and the bunch length and intensity are kept constant. In this case the luminosity and beamstrahlung vary linearly and quadratically with energy, respectively. At the lower end of the energy range, the beamstrahlung contribution to the BHSE decreases to a few $10^{-4}$. But on the other hand, electromagnetic deflections become rapidly stronger, dominating the bias below $400 \mathrm{GeV}$.

Another case of interest is to try maintaining a constant luminosity with energy. This may be useful for some specific physics studies. One way to achieve this is to scale $\beta_{x}$, $\beta_{y}$ and $\sigma_{z}$ linearly with energy, in order to maintain the mean relative beamstrahlung energy loss, $\delta$, constant. Within the planned range of ILC beam parameters [12], this is expected to be feasible for reductions by about a factor two at most. The corresponding results are shown on the right-hand side of Figure 12. In this case the bias on the Bhabha counting rate due to electromagnetic deflections becomes dominant under $300 \mathrm{GeV}$ and reaches several percent at low energy.

\section{Conclusion}

Beam-beam interactions at the collision point of linear colliders are the place of several well known quantum phenomena, such as the Bhabha scattering used to measure the most important interaction parameter: the luminosity.

In this study, it was shown, for the first time, that taking into account beam-beam interactions, the precise knowledge of the theoretical cross section is no longer sufficient to measure the luminosity with high precision (better than $10^{-3}$ ), because strong collective effects modify drastically the kinematics of the Bhabha process. The first changes come from beamstrahlung, which induces a perturbation of the initial state. A second set of transformations arises from electromagnetic deflections which modify the kinematical phase space of the scattered Bhabha events. Using the beam-beam interaction simulation tool GUINEA-PIG, it was estimated that the bias induced on the luminosity measurement at low angle is about -0.015 for the Nominal beam parameter set. Two thirds of this bias are due to beamstrahlung emissions.

Both beamstrahlung emissions and electromagnetic deflections vary with the bunch length, $\sigma_{z}$, the horizontal size, $\sigma_{x}$, and the energy of the collision, and hence also the resulting biases on the integrated luminosity. Reconstructing the luminosity spectrum 

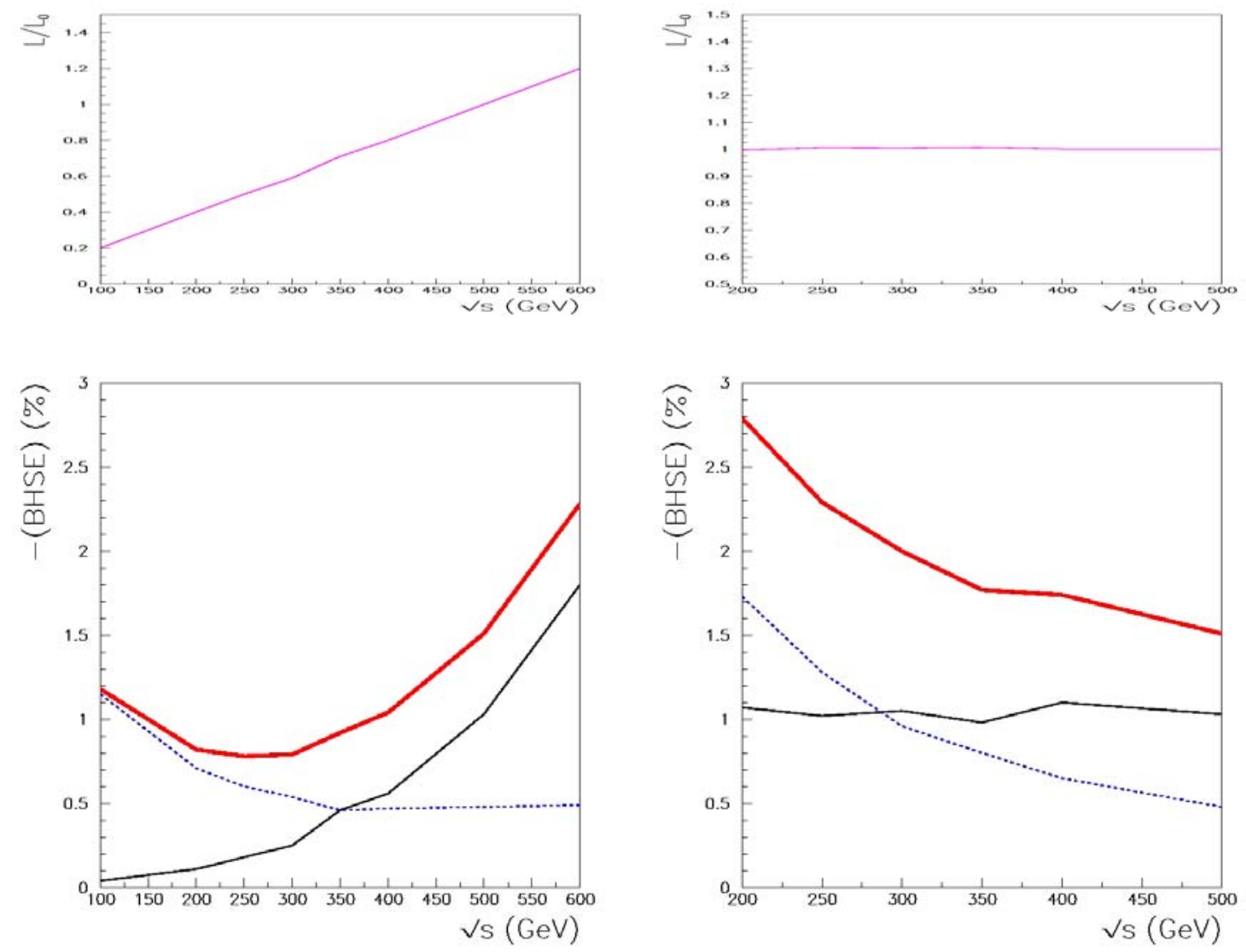

Figure 12: Bhabha suppression effect as a function of energy, keeping all optical parameters constant (left) and keeping the luminosity constant (right). The contributions due to beamstrahlung (thin line) and electromagnetic deflections (dashed line) are shown as well as the combined effect (top thick line). 
from the scattered Bhabha angles provides a good way to measure the amount of beamstrahlung, and thus to predict the corresponding contribution to the bias, because the electromagnetic deflections do not modify significantly this spectrum. The expected experimental precision on polar angle measurements should enable reaching an accuracy of $5 \cdot 10^{-4}$ for the mean value of the luminosity spectrum. This is enough to control the part of the luminosity bias from beamstrahlung at the $10^{-3}$ level. A fitting method, as the one suggested in [11], may allow to improve further on this. Once this is done, controlling $\sigma_{x}$ and $\sigma_{z}$ at the $20 \%$ level around the nominal values is enough to limit the remaining contribution to the luminosity bias, from the deflections, to $10^{-3}$.

Unlike the beamstrahlung emissions, no direct way to control experimentally the bias from the electromagnetic deflections is available from the data. Nevertheless, it can be noted that the disruption angle is proportional to the inverse of the transverse beam size. Hence, measurements of the beam angular divergence in the extraction line could provide a good way to monitor the luminosity bias from variations in $\sigma_{x}$. The dependence on $\sigma_{z}$ would however have to be controled in some other way. Further studies are needed on this point. Numerical simulations such as GUINEA-PIG [6] and CAIN [14] are essential estimation tools in all of this. A practical implementation to compute all these effects is available at [15] to this end.

In particular, physics running on the $\mathrm{Z}$ boson resonance is planned as an option in the ILC program (the GigaZ option). The accuracy on the luminosity is specified to be $10^{-4}$ in this case, while the bias from the electromagnetic deflections is at least a hundred times larger. Further more complete studies will then be particularly important, to explore all relevant dependencies and to devise appropriate data-driven correction methods.

\section{Acknowledgement}

This work is supported by the Commission of the European Communities under the $6^{\text {th }}$ Framework Programme "Structuring the European Research Area", contract number RIDS-011899. It has also benefited from useful discussions with several members of the FCAL R\&D Collaboration, in particular I. Bozovic-Jelisavcic, C. Grah, W. Lohman and M. Pandurovic.

\section{References}

[1] TESLA Technical Design Report, DESY 2001-011, March 2001.

[2] The Forward Calorimetry Group, "R\&D for the ILC-Detector: Instrumentation of the Very Forward Region", DESY PRC R\&D 02/01, April 2006.

http://www-zeuthen.desy.de/ILC/fcal/doc/PRC06/prc_rd_02-01_update_04-06.pdf

[3] S. Jadach, "Theoritical error of luminosity cross-section at LEP", june 2003. [hep$\mathrm{ph} / 0306083]$ 
[4] A. Stahl, "Luminosity Measurement via Bhabha Scattering: Precision Requirements for the Luminosity Calorimeter" LC-DET-2005-004, 2005.

[5] S. Jadach, W. Placzek, E. Richter-Was, B.F.L. Ward, Z. Was, "Upgrade of the Monte-Carlo program BHLUMI for Bhabha scattering at low angles to version 4.04", june 1996, CERN-TH/96-158. UTHEP-96-0601.

[6] D. Schulte, Ph. D. Thesis, University of Hamburg 1996. TESLA-97-08.

[7] OPAL Collaboration, G. Abbiendi et al., "Precision luminosity for Z0 line shape measurements with a silicon tungsten calorimeter", Eur.Phys.J.C14 (2000) 373-425. [hep-ex/9910066]

[8] ALEPH Collaboration, R. Barate et al., "Measurement of the Z resonance parameters at LEP", Eur.Phys.J.C14 (2000) 1-50.

[9] DELPHI Collaboration, P. Abreu et al., "Cross-sections and leptonic forward backward asymmetries from the Z0 running at LEP", Eur.Phys.J.C16 (2000) 371-405

[10] L3 Collaboration, M. Acciarri et al., "Measurements of cross-sections and forward backward asymmetries at the $\mathrm{Z}$ resonance and determination of electroweak parameters", Eur.Phys.J.C16 (2000) 1-40. [hep-ex/0002046]

[11] K. Mönig, "Measurement of the Differential Luminosity using Bhabha events in the Forward-Tracking region at TESLA", LC-PHSM-2000-60-TESLA, december 2000.

[12] International Linear Collider Reference Design Report, first draft, february 2007. http://media.linearcollider.org/rdr_draft_v1.pdf

[13] A. Djouadi, J. Lykken, K. Mönig, Y. Okada, M. Oreglia, S. Yamashita, "The Physics Case for the International Linear Collider", Frozen draft for the ACFA meeting in Beijing, February 2007.

http://www-zeuthen.desy.de/ILC/dcr_physics/dcr_beijing.pdf

[14] K. Yokoya, User's Manual of CAIN - Version 2.35, April 2003. http://lcdev.kek.jp/ yokoya/CAIN/cain235/CainMan235.pdf

[15] http://flc.web.lal.in2p3.fr/mdi/BBSIM/bbsim.html 